\title{
Weighted Performance comparison of DWT and LWT with PCA for Face Image Retrieval
}

\author{
J.Madhavan ${ }^{1}$, K.Porkumaran ${ }^{2}$ \\ ${ }^{1}$ Dept. of ECE, Adhiyamaan College of Engineering, Hosur, India \\ madhavece2006@yahoo.com \\ ${ }^{2}$ Dr. NGP college of Engineering and Technology, Coimbatore, India \\ porkumaran@gmail.com
}

\begin{abstract}
This paper compares the performance of face image retrieval system based on discrete wavelet transforms and Lifting wavelet transforms with principal component analysis (PCA). These techniques are implemented and their performances are investigated using frontal facial images from the ORL database. The Discrete Wavelet Transform is effective in representing image features and is suitable in Face image retrieval, it still encounters problems especially in implementation; e.g. Floating point operation and decomposition speed. We use the advantages of lifting scheme, a spatial approach for constructing wavelet filters, which provides feasible alternative for problems facing its classical counterpart. Lifting scheme has such intriguing properties as convenient construction, simple structure, integer-to-integer transform, low computational complexity as well as flexible adaptivity, revealing its potentials in Face image retrieval. Comparing to PCA and DWT with PCA, Lifting wavelet transform with PCA gives less computation and DWT-PCA gives high retrieval rate.. Especially 'sym2' wavelet outperforms well comparing to all other wavelets.
\end{abstract}

\section{KEYWORDS}

Discrete Wavelet Transform, Lifting Wavelet Transform, Principle component Analysis

\section{INTRODUCTION}

The main aim of Face image retrieval is to retrieve face images which are similar to a specific query face image in large face Databases. The retrieved face images can be used for many applications, such as photo management, Visual surveillance, Criminal face identification and searching specific faces from the internet etc. This retrieval task contains two types of target images. One is the face images with the same identity of the query face. The other is the face images which have appearance similar to the query face.

In Face image retrieval, wavelet approaches mainly include direct wavelet coefficients, DWT with PCA, DWT with LDA. Wavelet histogram and wavelet moment of image, etc. However, classical wavelet encounters some inherent limitations in image processing. First, classical construction relying heavily on frequency domain is basically unfit for spatial realization, inevitably losing desirable properties exclusively owned in spatial domain. Second, its floatingpoint operation makes it not only inefficient but also inaccurate when processing integer image coefficients [1]. Third, finding an ideal pre-processing method from classical view point is somewhat difficult, e.g. hard to seek a boundary extension method ensuring perfect reconstruction 
while simultaneously maintaining boundary continuity. Hence, traditional wavelet approaches, though effective in general case, may still result in reduced performance in face image retrieval.

Lifting scheme [2]-[5], a novel approach for constructing the so-called second-generation wavelet, provides feasible alternative for problems facing the classical first generation wavelet in image applications. Constructed entirely in spatial domain and based on the theory of different wavelet filter banks with perfect reconstruction, lifting scheme can easily build up a gradually improved multi-resolution analysis through iterative primal lifting and dual lifting. It turns out that lifting scheme outperforms the classical especially in effective implementation, such as convenient construction, in-place computation, lower computational complexity and simple inverse transform etc. We can also build wavelets with more vanishing moments and more smoothness, contributing to its flexible adaptivity and non-linearity.

Principal Component Analysis (PCA) method [6, 7], which is called Eigen faces in [8, 9] is widely used for dimensionality reduction and recorded a great performance in face image retrieval. PCA based approaches typically include two phases: training and classification. In the training phase, an Eigen space is established from the training samples using PCA method and the training face images mapped it for classification.

In this paper, we use DWT and lifting schemes to decompose Greyscale images into multilevel scale and wavelet coefficients, then further dimensionality reduction is done by using PCA with which we perform image feature extraction and similarity match by means of Euclidian distance method.. The retrieval performances are compared with those of its classical counterpart in terms of weighted retrieval accuracy and speed. The efficiency in terms of retrieval accuracy and speed is tested with various Face images using ORL database and the results outperform its classical counterpart.

The remainder of this paper is organized as follows. In section 2, we introduced the general structure of the proposed Face image Retrieval system. Section 3 provides the image decomposition using Discrete Wavelet Transform. Section 4 describes the Lifting Wavelet Transform scheme. Section 5 covers Feature Extraction using Principle Component Analysis. Section 6 explains Weighted Retrieval Accuracy. Section 7 describes the implementation and experimental results. Finally conclusions are offered in section 8 .

\section{General Structure of Proposed FaCe Retrieval SyStem}

Fig.1 shows the basic block diagram of Face image retrieval system. Our Proposed method compares the performance of face image retrieval using DWT-PCA and LWT-PCA with similarity matching by using Euclidian distance method.

We compared two methods, in first method; all train images decomposed using discrete wavelet transform. After DWT, we are taking only low frequency components (LL) of the image for further dimensionality reduction by using PCA. Then the final feature vectors of all the train images are stored in the database. Same process is done for query image. Finally first 5 similar face images are retrieved by using Euclidian distance method. In the second method, instead of DWT, we used Lifting wavelet transform for image decomposition and all other steps are same like first method. 


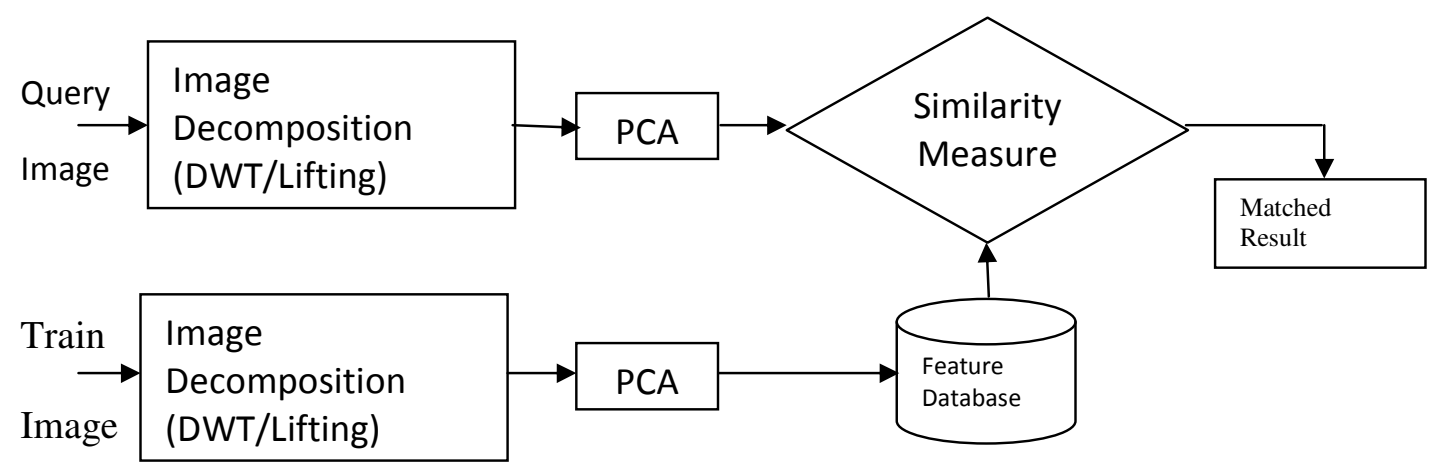

Fig.1 Block diagram of our proposed System

\section{DisCRETE WAVELET TRANSFORM}

The speciality of DWT [10] comparing to other transforms is time and frequency characteristics which application of it wildly in many different fields.

The flow chart of the Discrete wavelet transform sub band coding on the digital image is shown in figure 2 , here $\mathrm{L}$ refers to low frequency component, $\mathrm{H}$ refers to high frequency and the number 1 and 2 refer to the decomposition level of the Discrete wavelet transform. The result of the 2-D Discrete Wavelet Transform from level one to level three is shown in figure 3. The sub image LL is the low frequency component, it is the approximate sub image of the original image; the sub image $\mathrm{HL}$ is the component of the low frequency in horizontal direction and the high frequency in vertical direction, it manifests the horizontal edge of the original image; the sub image LH is the component of the high frequency in horizontal direction and the low frequency in vertical direction, it manifests the vertical edge of the original image; the sub image $\mathrm{HH}$ is the high frequency component, it manifests the oblique edge of the original image. It is shown that most energy of the original image is contained in the LL2 low frequency region. And the other region in the same size reflect edge feature of the image in different angles. The size of the low frequency sub-image is only $-2 \mathrm{n}$ power of 2 times of the source image if this transform was done for $\mathrm{n}$ times. The algorithm in this paper uses the low frequency sub images formed in the 2-D Discrete Wavelet Transform instead of the original face images, so that the dimension of the total population scatter matrix would reduced in the character extraction in the PCA method and consequently lessen the calculation amount.

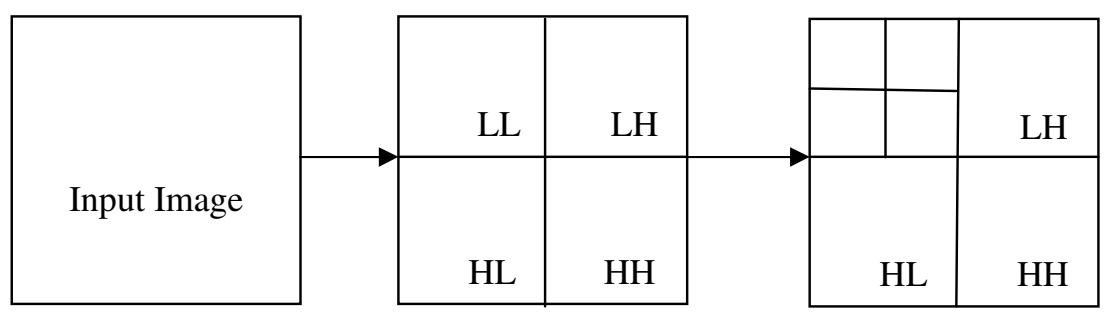

Fig.2 Flow chart of the DWT sub band coding 

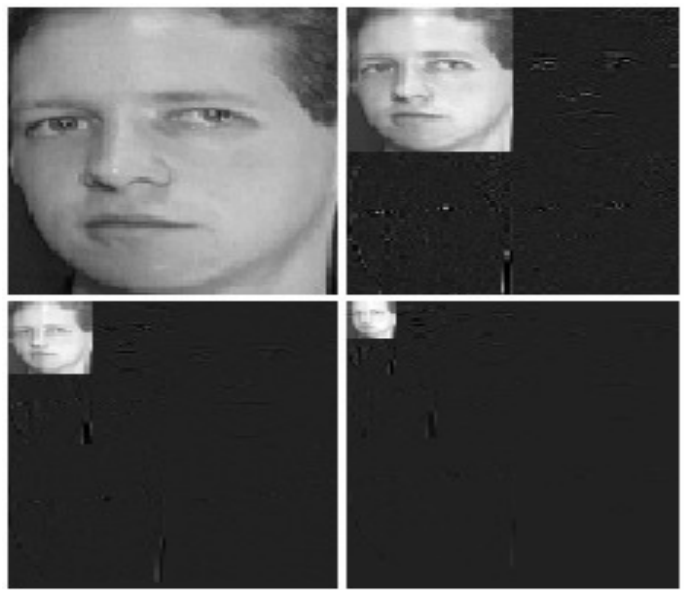

Fig.3. The result of the 2-D Discrete Wavelet Transform from level one to level three

\section{LIFTING WAVELETS TRANSFORM}

Any Discrete Wavelets Transform with Finite filters can be decomposed into a finite sequence of simple filtering steps, which are called the lifting steps [11]. Fig.4. shows the forward wavelet transform using lifting scheme. This decomposition related to a factorization of the polyphase matrix of wavelet or sub band filters into elementary matrices is described as follows. The polyphase representation of a discrete-time filter $h(z)$ is defined as

$$
h(z)=h_{\mathrm{e}}\left(\mathrm{z}^{2}\right)+\mathrm{z}^{-1} \mathrm{~h}_{\mathrm{o}}\left(\mathrm{z}^{2}\right)
$$

Where $h_{e}$ denotes the even coefficients, and $h_{o}$ denotes the odd coefficients:

$$
\begin{aligned}
& h_{e}(z)=\sum_{k} h_{2 k} z^{-k} \\
& h_{o}(z)=\sum_{k} h_{2 k+1} z^{-k}
\end{aligned}
$$

The low pass filter $\mathrm{h}(z)$ and High pass filter $g(z)$ can thus be represented by their poly phase matrix $\mathrm{P}(\mathrm{z})=\left[\begin{array}{ll}h_{e}(z) & g_{e}(z) \\ h_{o}(z) & g_{o}(z)\end{array}\right]$ and $\tilde{P}(z)$ can also be defined for the analysis filter analogously. The filters $\mathrm{h}_{e}(z), h_{o}(z), g_{e}(z)$ and $g_{o}(z)$, along with their analysis counterparts, are Laurent polynomials. As the set of all Laurent polynomials exhibits a commutative ring structure, within which polynomial division with remainder is possible, long division between Laurent polynomials is not a unique operation [12]. The Euclidean algorithm [12] can be used to decompose $P(z) \tilde{P}(Z)$ as

$$
\begin{aligned}
& P(z)=\prod_{i=1}^{m}\left[\begin{array}{cc}
1 & s_{i}(z) \\
0 & 1
\end{array}\right]\left[\begin{array}{cc}
1 & 0 \\
t_{i}(z) & 1
\end{array}\right]\left[\begin{array}{cc}
k & 0 \\
0 & 1 / k
\end{array}\right] \\
& \check{P}(z)=\prod_{i=1}^{m}\left[\begin{array}{cc}
1 & 0 \\
-s_{i}\left(z^{-1}\right. & 1
\end{array}\right]\left[\begin{array}{cc}
1 & -t_{i}\left(z^{-1}\right) \\
0 & 1
\end{array}\right]\left[\begin{array}{cc}
1 / K & 0 \\
0 & K
\end{array}\right]
\end{aligned}
$$

As this factorization is not unique, several pairs of $\left\{s_{i}(z)\right\}$ and $\left\{t_{i}(z)\right\}$ filters are admissible; However, in case of DWT implementation, all possible choices are equivalent. 


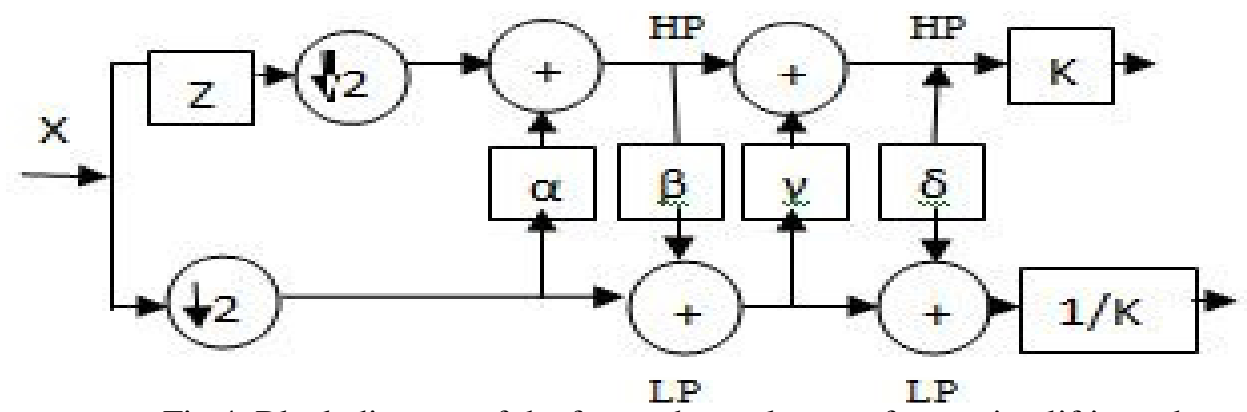

Fig.4. Block diagram of the forward wavelet transform using lifting scheme

\section{PRINCIPLE COMPONENT ANALYSIS}

Principal component analysis [13] is classical method used in statistical pattern recognition and signal processing for dimensionality reduction and feature extraction.

Every test image can be transformed to low dimensional feature vector to be projected onto the eigenface space which was obtained from the training set. This feature vector can then be compared with the set of feature vectors obtained from the training set. The face classifier can use different distance measures such as Euclidean distance or cosine distance. The PCA algorithm can be detailed as follows:

Let the training set of face images be $\Gamma_{1}, \Gamma 2, \ldots, \Gamma \mathrm{M}$ then the average of the set is defined by

$$
\Psi=\frac{1}{M} \sum_{n=1}^{M} \Gamma_{n}
$$

Each face differs from the average by the vector

$$
\Phi_{i}=\Gamma_{i}-\Psi
$$

This set of very large vectors is then subject to PCA, which seeks a set of $M$ orthonormal Matrix $\mathrm{C}$ can be defined as

$$
C=\frac{1}{M} \sum_{n=1}^{M} \Phi_{n} \Phi_{n}^{T}=\mathrm{A} \mathrm{A}^{T}
$$

Vectors, Um, which best describes the distribution of the data. Then the covariance

Where the matrix $A=\left[\begin{array}{ll}\Phi_{1} & \Phi_{2} \ldots . . \Phi_{\mathrm{M}}\end{array}\right]$. The covariance matrix $C$; however is $N 2 \times N 2$ real Symmetric matrix, and determining the $N 2$ eigenvectors and eigenvalues is an intractable task for typical image sizes. We need a computationally feasible method to find these eigenvectors.

Consider the eigenvectors $\mathrm{v}_{i}$ of $\mathrm{A}^{\mathrm{T}} \mathrm{A}$ such that

$$
\mathrm{A}^{T} A v_{\mathrm{i}}=\mu_{\mathrm{i}} \mathrm{v}_{\mathrm{i}}
$$

Premultiplying both sides by $\mathrm{A}$, we have

$$
\mathrm{AA}^{\mathrm{T}} \mathrm{Av}_{\mathrm{i}}=\mu_{\mathrm{i}} \mathrm{Av}_{i}
$$

Where we see that Avi are the eigenvectors and $\mu_{\mathrm{i}}$ are the eigenvalues of $C=\mathrm{AA}^{\mathrm{T}}$. Following these analysis, we construct the $M \times M$ matrix $L=A^{T} A$, where $L m n=\Phi_{m}^{T} \Phi_{n}$, and find the $M$ eigenvectors, $\mathrm{v}_{i}$, of $L$. These vectors determine linear combinations of the $M$ training set face images to form the eigenfaces $\mathrm{U}_{\mathrm{I}}$. 


$$
U_{I}=\sum_{k=1}^{M} V_{I K} \Phi_{\mathrm{k}}, \quad \mathrm{I}=1 \ldots \mathrm{M}
$$

With this analysis, the calculations are greatly reduced; from the order of the number of pixels in the images $(N 2)$ to the order of the number of images in the training set $(M)$. The associated eigenvalues allow us to rank the eigenvectors according to their usefulness in characterizing the variation among the images.

A new face image $(\Gamma)$ is transformed into its eigenface components (projected onto "face space") by a simple operation,

$$
w_{k}=U_{k}^{T}(\Gamma-\Psi)
$$

For $k=1, \ldots, M^{\prime}$. The weights form a projection vector,

$$
\Omega^{T}=\left[w_{1}, w_{2}, \ldots . . w_{T}\right]
$$

Describing the contribution of each eigenface in representing the input face image, treating the eigenfaces as a basis set for face images. The projection vector is then used to find which of a number of predefined face classes that best describes the face.

\section{WEIGHTED RETRIEVAL ACCURACY}

We used the new method called, Weighted Retrieval Accuracy for face image retrieval accuracy Calculations. Let us assume that A, B, C, D, E are First five retrieved Face images respectively. Assign weights for each retrieved images. Here we given more weight for first priority retrieved image and least weight for fifth retrieved image. The weighted values 35, 30, 20, 10, 5 are first five retrieved images respectively.

Then the weighted retrieval accuracy calculated as follows.

$$
\mathrm{WRA}=35 * \mathrm{~A}+30 * \mathrm{~B}+20 * \mathrm{C}+10 * \mathrm{D}+5 * \mathrm{E}
$$

Here WRA stands for Weighted Retrieval Accuracy.

\section{SIMULATION RESULTS AND DISCUSSION}

We performed our simulations on the ORL database [14] with the platform of MAT Lab 7.8. This database consists of 400 face images attained from 40 people. Each people have 10 images of different expression, poses and different lighting conditions. The resolution rate of each image is 112 X92 and the gray scale is 256 . Out of the 400 images in the ORL face database, first 399 were selected for training and each images were used for testing. Fig.5 shows sample images from the database.

Our experiment gives the performance comparison results of Face image retrieval using DWTPCA and LWT-PCA both in level1 and level2 decompositions. Here feature extraction is done by using DWT/LWT and further dimension reduction is done by using PCA. 


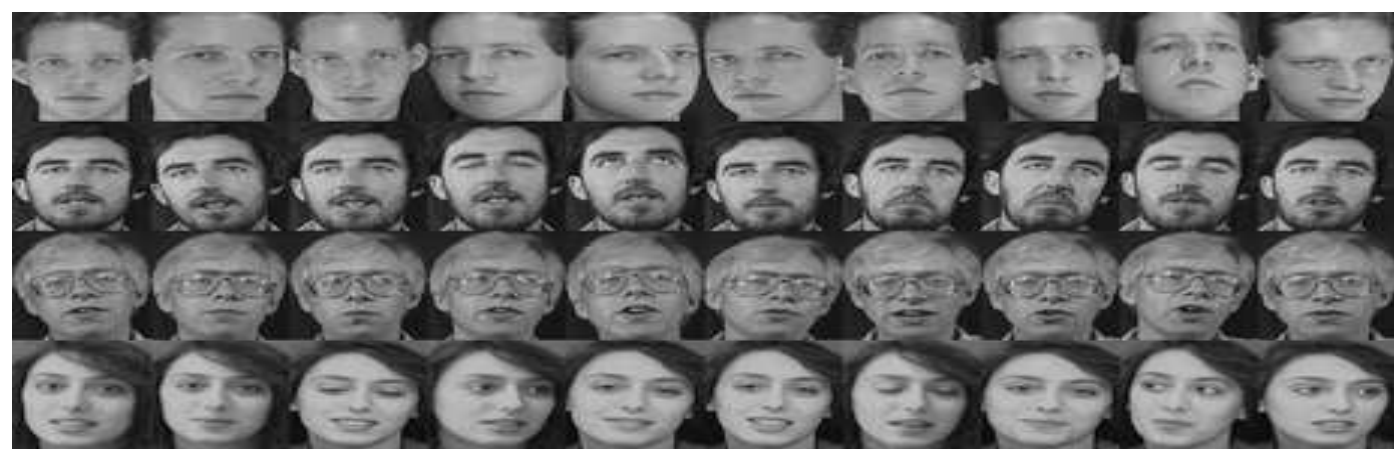

Fig.5 ORL Database sample images

The Similarity matching is achieved by using Euclidian distance method. Fig.6.a, b shows the Query face image and the retrieved face images of 5 most similar images illustrated respectively. The weighted Average Retrieval accuracy and Average Standard deviations class wise weighted retrieval accuracy and standard deviation using DWT-PCA and LWT-PCA for both level1 and level 2 decomposition Table is shown in Table 1 and Total average Retrieval accuracy and total average standard deviation comparison table is shown in Table.2.

The comparison table 1 and 2 shows that Weighted Retrieval Accuracy and Standard devastations are inversely proportional. I.e. Weighted retrieval accuracy increases then its standard deviation decreases and vice versa. Fig.7,8 shows the performance plots of Weighted Retrieval Accuracy for each Class of DWT-PCA and LWT-PCA with level1 and level2 decompositions respectively. Fig.9, 10 shows the performance plots of Standard Deviation for each Class of DWT-PCA and LWT-PCA with level1 and level2 decompositions respectively.
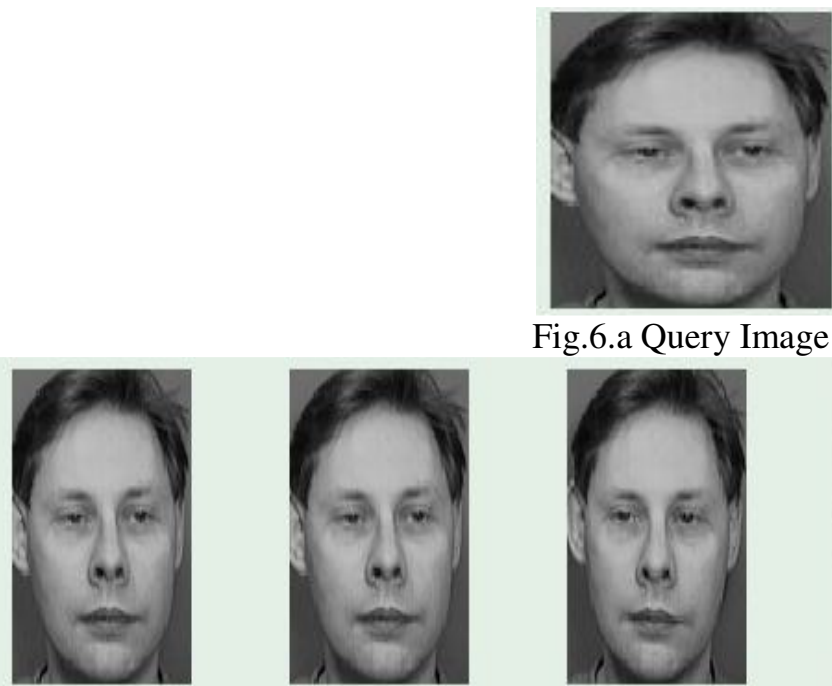

Fig.6.a Query Image
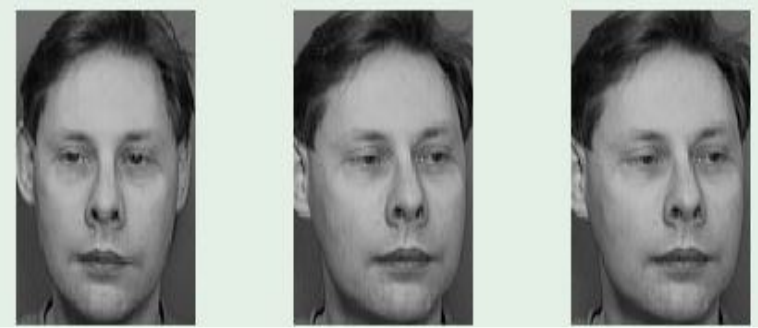

Fig.6.b. Retrieved Images 


\begin{tabular}{|c|l|l|}
\hline Methods & $\begin{array}{l}\text { Average } \\
\text { Weighted } \\
\text { Retrieval } \\
\text { Accuracy } \\
\%\end{array}$ & $\begin{array}{l}\text { Average } \\
\text { Standard } \\
\text { Deviation }\end{array}$ \\
\hline DWT-PCA(Level1) & 91.475 & 8.479 \\
\hline DWT-PCA(Level2) & 92.575 & 7.93 \\
\hline LWT-PCA(Level1) & 90.85 & 8.68 \\
\hline LWT-PCA(Level2) & 90.45 & 8.875 \\
\hline
\end{tabular}

Table2: Weighted Average total Retrieval Accuracy and Total average Standard Deviation.

\section{CONCLUSION}

In this paper performance comparison of two methods named, Face retrieval using DWT with PCA and Face retrieval using LWT with PCA are proposed. Comparing to these two methods DWT with PCA using 'sym2' wavelet with Level2 method outperforms well for Weighted Retrieval Accuracy. LWT with PCA method gives less elapsed time comparing to DWT with PCA method. Among these two methods the level of decomposition increases then the dimensions of feature vectors reduced. So it decreases the elapsed time but the weighted retrieval accuracy decreased. 


\begin{tabular}{|c|c|c|c|c|c|c|c|c|}
\hline \multirow{3}{*}{$\begin{array}{l}\text { Class } \\
\text { Name }\end{array}$} & \multicolumn{4}{|c|}{ DWT-PCA } & \multicolumn{4}{|c|}{ LWT-PCA } \\
\hline & \multicolumn{2}{|c|}{$\begin{array}{l}\text { Retrieval } \\
\text { Accuracy \% }\end{array}$} & \multicolumn{2}{|c|}{ Standard Deviation } & \multicolumn{2}{|c|}{$\begin{array}{l}\text { Retrieval } \\
\text { Accuracy \% }\end{array}$} & \multicolumn{2}{|c|}{ Standard Deviation } \\
\hline & Level1 & Level2 & Level1 & Level2 & Level1 & Level2 & Level1 & Level2 \\
\hline 1 & 99 & 99 & 2 & 2 & 99 & 99 & 2 & 2 \\
\hline 2 & 84.5 & 87 & 14.04457 & 12.49 & 82.5 & 83 & 12.5 & 13.0767 \\
\hline 3 & 94 & 96.5 & 7.348469 & 4.5 & 94 & 93.5 & 7.348469 & 7.433034 \\
\hline 4 & 95.5 & 95.5 & 6.873864 & 6.873864 & 95.5 & 97 & 6.873864 & 5.09902 \\
\hline 5 & 97 & 97 & 5.09902 & 5.09902 & 97 & 99.5 & 5.09902 & 1.5 \\
\hline 6 & 100 & 100 & 0 & 0 & 99 & 98 & 2 & 3.316625 \\
\hline 7 & 98.5 & 99.5 & 3.201562 & 1.5 & 98 & 84.5 & 3.316625 & 22.07374 \\
\hline 8 & 88.5 & 90.5 & 14.6714 & 19.16377 & 84.5 & 85.5 & 22.07374 & 16.5 \\
\hline 9 & 93 & 94 & 11.87434 & 12.20656 & 93 & 97 & 11.87434 & 4.582576 \\
\hline 10 & 98.5 & 98.5 & 3.201562 & 3.201562 & 98 & 99.5 & 3.316625 & 1.5 \\
\hline 11 & 99.5 & 99.5 & 1.5 & 1.5 & 99.5 & 99.5 & 1.5 & 1.5 \\
\hline 12 & 95.5 & 95.5 & 5.678908 & 5.678908 & 95.5 & 95.5 & 5.678908 & 5.678908 \\
\hline 13 & 99 & 99 & 2 & 2 & 98.5 & 98.5 & 2.291288 & 2.291288 \\
\hline 14 & 81 & 82.5 & 14.45683 & 14.87447 & 79.5 & 79 & 14.7394 & 14.45683 \\
\hline 15 & 88.5 & 89 & 13.42572 & 12.60952 & 88.5 & 88.5 & 13.42572 & 13.42572 \\
\hline 16 & 97.5 & 98 & 4.609772 & 3.316625 & 97.5 & 96.5 & 4.609772 & 5.024938 \\
\hline 17 & 93 & 93 & 19.39072 & 19.39072 & 92.5 & 92.5 & 19.39716 & 19.39716 \\
\hline 18 & 85 & 85 & 11.61895 & 11.61895 & 84 & 84.5 & 12 & 11.5 \\
\hline 19 & 99 & 99 & 2 & 2 & 98.5 & 99 & 2.291288 & 2 \\
\hline 20 & 100 & 100 & 0 & 0 & 100 & 100 & 0 & 0 \\
\hline 21 & 72.5 & 76 & 8.732125 & 9.69536 & 71.5 & 71 & 9.5 & 9.433981 \\
\hline 22 & 99.5 & 99.5 & 1.5 & 1.5 & 99.5 & 99.5 & 1.5 & 1.5 \\
\hline 23 & 85.5 & 91.5 & 15.88238 & 12.05197 & 84 & 83 & 13.37909 & 15.36229 \\
\hline 24 & 98 & 99 & 2.44949 & 2 & 98.5 & 97.5 & 2.415229 & 3.354102 \\
\hline 25 & 97 & 97 & 4.582576 & 4.582576 & 97 & 97 & 4.582576 & 4.582576 \\
\hline 26 & 89.5 & 89 & 18.63465 & 18.81489 & 89 & 89 & 18.54724 & 18.54724 \\
\hline 27 & 94 & 96 & 5.830952 & 4.358899 & 92.5 & 91.5 & 7.826238 & 8.674676 \\
\hline 28 & 99.5 & 99.5 & 1.5 & 1.5 & 99.5 & 99.5 & 1.5 & 1.5 \\
\hline 29 & 91.5 & 92.5 & 10.96586 & 10.78193 & 91 & 87.5 & 10.67708 & 12.69843 \\
\hline 30 & 92.5 & 94.5 & 19.39716 & 13.5 & 92 & 92 & 19.51922 & 19.51922 \\
\hline 31 & 97.5 & 98.5 & 6.020797 & 4.5 & 97.5 & 97.5 & 6.020797 & 6.020797 \\
\hline 32 & 99 & 100 & 2 & 0 & 98.5 & 98.5 & 2.415229 & 2.291288 \\
\hline 33 & 62 & 66 & 13.45362 & 13.74773 & 59.5 & 59 & 14.23025 & 14.10674 \\
\hline 34 & 83 & 85 & 15.0333 & 10.95445 & 82.5 & 82 & 15.67907 & 14.3527 \\
\hline 35 & 100 & 100 & 0 & 0 & 100 & 100 & 0 & 0 \\
\hline 36 & 91 & 90.5 & 10.19804 & 10.11187 & 90.5 & 90.5 & 10.65885 & 10.11187 \\
\hline 37 & 88.5 & 93.5 & 10.01249 & 5.024938 & 87 & 85.5 & 10.32796 & 11.05667 \\
\hline 38 & 75.5 & 76.5 & 10.59481 & 11.84272 & 75 & 74.5 & 10.27402 & 10.11187 \\
\hline 39 & 68 & 70.5 & 20.63977 & 22.96193 & 68 & 67.5 & 21.75623 & 20.40221 \\
\hline 40 & 88.5 & 90 & 18.71497 & 19.10497 & 86.5 & 85.5 & 19.44365 & 19.03287 \\
\hline AVG & 91.475 & 92.575 & 8.479 & 7.93 & 90.85 & 90.45 & 8.68 & 8.875 \\
\hline
\end{tabular}

Table2: The comparison of Average Retrieval Accuracy and Average Standard Deviation of Each Class. 


\section{REFERENCES}

[1] R. Calderbank, I. Daubechies, W. Sweldens and Y.Boon-Lock.Wavelet transform that map integers to integers. Journal of Applied and Computational Harmonic Analysis .5(3), 332-369, 1998.

[2] I. Daubechies and W. Sweldens. Factoring wavelet transforms into lifting steps. Journal of Fourier analysis and Applications. 4(3), 245-267, 1998.

[3] W. Sweldens. The lifting scheme: a custom-design construction of biorthogonal wavelets. Journal of Applied and Computational Harmonic Analysis. 3(2), 186-200, 1995.

[4] G. Uytterhoeven, D. Roose and A. Bultheel. Wavelet transform using the lifting scheme. Report

[5] R. Calderbank, I. Daubechies, W. Sweldens and Y.Boon-Lock.Wavelet transform that map integers to integers. Journal of Applied and Computational Harmonic Analysis .5(3), 332-369, 1998

[6] M. Kirby and L. Sirovich, Application of the Karhunen-Loeve Procedure for the Characterization of Human Faces, IEEE PAMI, Vol. 12 (1990) 103-108.

[7] M. Turk and A. Pentland, Eigenfaces for Recognition, Journal of Cognitive Neuroscience, Vol. 3, (1991) 71-86.

[8] A. Pentland, B. Moghaddam, and T. Starner. View based and modular eigenspaces for face recognition In Proceedings of the 1994 Conference on Computer Vision and Pattern Recognition, pages 84-91, Seattle, WA, 1994. IEEE Computer Society.

[9] Hong Wang, Su Yang, Wei Liao, An Improved PCA Face Recognition Algorithm Based on the Discrete Wavelet Transform and the Support Vector Machines, International Conference on Computational Intelligence and Security Workshops 308-311,2007.

[10] Chon Fong Wong, Jianke Zhu, Mang I Vai, eng Un Mak and Weikou Ye, Face Retrieval with Relevance Feedback Using Lifting Wavelets Features.

[11] Daubechies, I. and W. Sweldens. FFactoring wavelet transforms into lifting steps,"J. Fourier Anal. Appl., Vol. 4, Nr. 3, 1998.

[12] Alaa Eleyan and Hasan Demirel, PCA and LDA Based Face Recognition Using Feed forward Neural Network Classifier, MRCS 2006, LNCS 4105, pp. 199 - 206, 2006.

[13] 18. AT \& T Laboratories Cambridge. The ORL Database of faces. Http://www.camorl.co.uk/face database.html. 